\title{
Transporte de enxofre para as raízes de soja em três solos de Minas Gerais ${ }^{(1)}$
}

\author{
Davi José Silva(2), Víctor Hugo Alvarez Venegas ${ }^{(3)}$ e Hugo Alberto Ruiz ${ }^{(3)}$
}

\begin{abstract}
Resumo - Com o objetivo de avaliar a contribuição do fluxo em massa e da difusão no transporte de $\mathrm{S}$ para a superfície das raízes de soja, conduziu-se um ensaio em casa de vegetação, com amostras de três solos dos municípios de Viçosa, Paracatu e Lassance. Essas amostras apresentavam, respectivamente, $5,0,1,2$ e $1,4 \mathrm{mg} \mathrm{dm}^{-3}$ de $\mathrm{S}$ disponível, obtidos pelo extrator $\mathrm{Ca}\left(\mathrm{H}_{2} \mathrm{PO}_{4}\right)_{2}, 500 \mathrm{mg} \mathrm{L}^{-1}$ de $\mathrm{P}$ em $\mathrm{HOAc}$ $2 \mathrm{~mol} \mathrm{~L}^{-1}$. O experimento correspondeu a um fatorial $3 \times 5$, sendo três solos e cinco doses de $\mathrm{S}(0,20$, 40, 80 e $160 \mathrm{mg} \mathrm{dm}^{-3}$ ), dispostos em blocos casualizados, com quatro repetições. A umidade, controlada pelo uso de um tensiômetro por vaso, foi mantida próximo a -10 kPa durante todo o ensaio. O fluxo em massa foi o principal mecanismo de transporte de $\mathrm{S}$ para a superfície das raízes da soja. Quando a concentração de $\mathrm{S}$ na solução do solo foi alta, esse mecanismo supriu quantidades de $\mathrm{S}$ superiores às absorvidas pela planta. A contribuição da difusão para o suprimento de $\mathrm{S}$ ocorreu apenas em baixa concentração desse nutriente na solução do solo.
\end{abstract}

Termos para indexação: Glycine max, transporte de nutrientes, difusão, fluxo de massa, solução do solo.

\section{Sulphur transport toward soybean roots in three soils from Minas Gerais State, Brazil}

\begin{abstract}
A greenhouse experiment was carried out to evaluate the mass flow and diffusion contributions on sulphur transport toward soybean roots in soil surface samples $(0-20 \mathrm{~cm})$ of three soils collected in Viçosa, Paracatu and Lassance, Minas Gerais State, Brazil. Originally, the soil samples presented 5.0, 1.2 and $1.4 \mathrm{mg} \mathrm{dm}^{-3}$ of available $\mathrm{S}$, respectively, obtained by extraction with a $\mathrm{Ca}\left(\mathrm{H}_{2} \mathrm{PO}_{4}\right)_{2}$ solution containing $500 \mathrm{mg} \mathrm{L}^{-1}$ of $\mathrm{P}$ in HOAc $2 \mathrm{~mol} \mathrm{~L}^{-1}$ solution. A $3 \times 5$ factorial layout was used with three soils and five $S$ rates $\left(0,20,40,80\right.$ and $\left.160 \mathrm{mg} \mathrm{dm}^{-3}\right)$, disposed in a randomized complete block design, with four replications. Tensiometers controlled the soil water potential in each pot and the potential was maintained around $-10 \mathrm{kPa}$. The mass flow was the main mechanism of $\mathrm{S}$ transport to soybean roots. At high concentration in soil solution, the quantities supplied by mass flow were higher than those required by plants. Diffusion process only contributed to transport at low $\mathrm{S}$ concentration in soil solution.
\end{abstract}

Index terms: Glycine max, nutrient transport, diffusion, mass flow, soil solution.

\section{Introdução}

O transporte dos íons presentes na solução do solo até as raízes dos vegetais é feito por dois mecanismos: fluxo em massa e difusão. O fluxo em mas-

\footnotetext{
(1) Aceito para a publicação em 9 de novembro de 2001.

Extraído da tese de doutorado apresentada pelo primeiro autor à Universidade Federal de Viçosa (UFV), Viçosa, MG. Parcialmente financiado pelo CNPq.

${ }^{(2)}$ Embrapa-Centro de Pesquisa Agropecuária do Trópico SemiÁrido, Caixa Postal 23, CEP 56300-970 Petrolina, PE. E-mail: davi@cpatsa.embrapa.br

${ }^{(3)}$ UFV, Dep. de Solos, Av. P. H. Rolfs, s/no, CEP 36571-000 Viçosa, MG. Bolsista do CNPq. E-mail: vhav@mail.ufv.br, hruiz@mail.ufv.br
}

sa está associado ao gradiente de potencial hídrico provocado pela absorção de água pelas plantas. Desse modo, a concentração do íon na solução do solo e a taxa de transpiração do vegetal determinam a quantidade transportada por esse mecanismo. A difusão, por sua vez, é causada pelo movimento térmico ao acaso dos íons em direção à raiz, em virtude do gradiente de concentração gerado na superfície radicular (Barber, 1984).

Além desses dois mecanismos, a intercepção radicular também é responsável pelo suprimento de nutrientes às plantas. Barber (1984), entretanto, considera praticamente desprezível a sua participação no suprimento de $\mathrm{S}$ e Patel et al. (1984) questionam o seu verdadeiro significado, uma vez que o efeito 
do alongamento da raiz está incluído nos modelos de difusão mais recentes.

O fluxo em massa é o principal mecanismo de suprimento de $\mathrm{S}$ às raízes. Barber et al. (1963), utilizando uma auto-radiografia, demonstraram que o ${ }^{35} \mathrm{~S}$ aplicado uniformemente em todo o volume de solo do vaso moveu-se juntamente com a água para a superfície das raízes de milho. Em solos com alta concentração de sais, Ende (1973) observou que o fluxo em massa é capaz de suprir sulfato, cloreto, $\mathrm{Ca}, \mathrm{Mg}$ e $\mathrm{Na}$ em concentrações maiores que as absorvidas pelas raízes do tomateiro. Em plantas de soja, Xu et al. (1996a, 1996b) constataram que o fluxo em massa transportou quantidades de $\mathrm{Ca}, \mathrm{Mg}$, $\mathrm{Cu}$ e $\mathrm{Zn}$ muito acima das absorvidas pela planta, enquanto as quantidades de $\mathrm{P}, \mathrm{K}, \mathrm{Fe}$ e $\mathrm{Mn}$ transportadas por esse mesmo mecanismo não foram suficientes para satisfazer a demanda, e foram complementadas pela difusão. Gregory et al. (1979) também detectaram uma contribuição do fluxo em massa quatro vezes maior que a quantidade de $S$ absorvida por plantas de trigo cultivadas em um solo com altos teores deste nutriente. Mesmo em um solo ácido e bastante intemperizado, mas com suprimento adequado de S, Prenzel (1979) verificou que o fluxo em massa transportou uma quantidade de $S$ suficiente para acumular-se em torno das raízes de faia (Fagus sylvatica). Em plantas de milho, o fluxo em massa foi o principal mecanismo de transporte de $\mathrm{S}$, promovendo também o seu acúmulo na superfície da raiz (Silva et al., 1998).

Desse modo, em condições de elevada concentração de $\mathrm{S}$ na solução do solo em face da fertilidade natural do solo ou da aplicação de fertilizantes, o fluxo em massa promove o suprimento do nutriente em quantidade suficiente para as plantas. Em solos mais intemperizados, com baixa concentração de nutrientes na solução do solo, o fluxo em massa pode ser insuficiente para satisfazer a demanda da planta.

Este trabalho teve por objetivo avaliar a contribuição dos mecanismos de fluxo em massa e de difusão para o suprimento de $S$ às raízes de soja, em três solos de Minas Gerais.

\section{Material e Métodos}

Foram utilizadas amostras coletadas na camada de 0 a $20 \mathrm{~cm}$ de três solos (Tabela 1) dos municípios de Viçosa, Paracatu e Lassance, MG. As amostras, depois de secadas ao ar, foram passadas em peneira de $4 \mathrm{~mm}$, divididas em subamostras de $5 \mathrm{dm}^{3}$, e receberam, em seguida, uma mistura dos carbonatos de cálcio e de magnésio na relação molar de 4:1. A quantidade de corretivo aplicada baseouse nos teores de argila e de cátions trocáveis de cada amostra de solo (Comissão de Fertilidade do Solo do Estado de Minas Gerais, 1989). Após a calagem, as amostras foram acondicionadas em sacolas de plástico e incubadas, mantendo-se a umidade em torno de $-10 \mathrm{kPa}$ por um período de 30 dias. O solo Lassance não recebeu corretivo e tampouco foi incubado, uma vez que apresentava eleva-

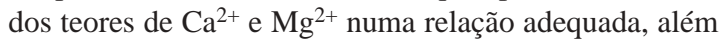
de seu teor de $\mathrm{Al}^{3+}$ estar abaixo do limite de detecção pelo método empregado.

Após a secagem e homogeneização, as amostras de solo receberam os tratamentos com S: 0, 20, 40, 80 e $160 \mathrm{mg} \mathrm{dm}^{-3}$ de $\mathrm{S}$, além de doses de nivelamento de $\mathrm{N}$ $\left(50 \mathrm{mg} \mathrm{dm}^{-3}\right), \mathrm{P}\left(450 \mathrm{mg} \mathrm{dm}^{-3}\right.$ nos solos de Viçosa e Paracatu e $300 \mathrm{mg} \mathrm{dm}^{-3}$ no solo de Lassance) e K (150 $\mathrm{mg} \mathrm{dm}^{-3}$ menos o teor de $\mathrm{K}$ disponível), fornecidos pelos sais $\mathrm{K}_{2} \mathrm{SO}_{4},\left(\mathrm{NH}_{4}\right)_{2} \mathrm{SO}_{4}, \mathrm{MgSO}_{4}, \mathrm{NH}_{4} \mathrm{H}_{2} \mathrm{PO}_{4}$, $\mathrm{KH}_{2} \mathrm{PO}_{4}$ e $\mathrm{Ca}\left(\mathrm{H}_{2} \mathrm{PO}_{4}\right)_{2}$.

A umidade foi ajustada gravimetricamente para $-10 \mathrm{kPa}$. As amostras, umedecidas e colocadas em sacos de plástico fechados, foram mantidas por sete dias nessas condições, para obter maior uniformidade na distribuição de umidade do material.

O experimento, conduzido em casa de vegetação entre dezembro de 1991 e janeiro de 1992, correspondeu a um fatorial 3 x 5 (três solos e cinco doses de $S$ ), e os tratamentos foram dispostos em blocos casualizados, com quatro repetições.

A unidade experimental foi constituída por um vaso de plástico com 4,5 dm³ de solo. Após o período de uniformização da umidade, as amostras de solo foram acondicionadas nos vasos, e foram plantadas, em seguida, cinco sementes por vaso de soja (Glycine max (L.) Merrill, variedade UFV-2). Após a emergência, efetuou-se o desbaste, deixando-se três plantas por vaso.

A umidade, controlada pelo uso de um tensiômetro por vaso (Ruiz, 1986), foi mantida, com água destilada, próximo a $-10 \mathrm{kPa}$, durante o período experimental. $\mathrm{O}$ volume de água evaporada no decorrer do experimento foi estimado com vasos contendo solo sem planta. Em cada bloco foram acrescentados três vasos sem planta, um para cada solo em estudo.

Para reduzir as perdas de água por evaporação, a superfície do solo de cada vaso foi coberta com uma camada de esferas de isopor com diâmetro de 2 a $7 \mathrm{~mm}$.

A quantidade de água transpirada pelas plantas foi determinada subtraindo-se do volume total aplicado em cada vaso, a quantidade evaporada. 
Semanalmente, foram aplicados a cada unidade experimental $25 \mathrm{mg} \mathrm{dm}^{-3}$ de $\mathrm{N}$, fornecidos como $\left(\mathrm{NH}_{2}\right)_{2} \mathrm{CO}$ em forma de solução. Os micronutrientes também foram fornecidos em solução, de modo que fossem adicionados a cada $\mathrm{dm}^{3}$ de solo um total de $0,813 \mathrm{mg}$ de $\mathrm{B}\left(\mathrm{H}_{3} \mathrm{BO}_{3}\right)$, 3,664 mg de $\mathrm{Mn}\left(\mathrm{MnCl}_{2} \cdot 4 \mathrm{H}_{2} \mathrm{O}\right), 4 \mathrm{mg}$ de $\mathrm{Zn}\left(\mathrm{ZnCl}_{2}\right)$, $1,329 \mathrm{mg}$ de $\mathrm{Cu}\left(\mathrm{CuCl}_{2} \cdot 2 \mathrm{H}_{2} \mathrm{O}\right), 1,556 \mathrm{mg}$ de $\mathrm{Fe}$ $\left(\mathrm{FeCl}_{3} \cdot 6 \mathrm{H}_{2} \mathrm{O}\right)$ e $0,15 \mathrm{mg}$ de $\mathrm{Mo}\left(\mathrm{Na}_{2} \mathrm{MoO}_{4} \cdot 2 \mathrm{H}_{2} \mathrm{O}\right)$ (Alvarez Venegas, 1974). Essas doses foram igualmente parceladas em quatro aplicações. Como o solo de Lassance apresentava elevada concentração de Mn (Tabela 1), as plantas cultivadas neste solo começaram a exibir sintomas de toxidez de manganês. Este nutriente foi então excluído da solução a partir da terceira aplicação, nas unidades experimentais cultivadas no solo de Lassance. $\mathrm{Na}$ última cobertura nitrogenada, foram fornecidos a todas as unidades experimentais $50 \mathrm{mg} \mathrm{dm}^{-3} \mathrm{de} \mathrm{Ne} 110 \mathrm{mg} \mathrm{dm}^{-3}$ de $\mathrm{P}$ na forma de solução de $\mathrm{NH}_{4} \mathrm{H}_{2} \mathrm{PO}_{4}$.

Quarenta dias após o plantio, a parte aérea e as raízes de soja foram colhidas e secadas em estufa de ventilação forçada a $70^{\circ} \mathrm{C}$, onde permaneceram até o peso se manter constante. Após a secagem, o material vegetal foi moído e mineralizado por digestão nítrico-perclórica, e no extrato obtido procedeu-se à dosagem do $\mathrm{S}$ por turbidimetria (Blanchar et al., 1965).
Amostras de solo de todas as unidades experimentais foram secadas e passadas em peneira de $2 \mathrm{~mm}$. Em seguida, foram tomadas subamostras para a extração da solução do solo. A solução do solo foi obtida pelo método do extrato da pasta de saturação (Embrapa, 1997), e sua concentração em S foi dosada por turbidimetria (Chesnin \& Yien, 1950).

A contribuição do fluxo em massa para o transporte de $\mathrm{S}$ foi determinada e considerou-se a quantidade de água transpirada pelas plantas e a concentração do nutriente existente no extrato da pasta de saturação (Oliver \& Barber, 1966).

O S transportado por difusão foi calculado subtraindo-se do $\mathrm{S}$ total acumulado na planta o valor correspondente ao $\mathrm{S}$ transportado por fluxo em massa.

\section{Resultados e Discussão}

De maneira geral, as quantidades de $\mathrm{S}$ transportadas por fluxo em massa foram superiores às absorvidas pelas plantas. Isto pode ser observado pela quantidade de $\mathrm{S}$ transportada por fluxo em massa e pelo seu conteúdo nas plantas de soja (Tabela 2). Nos tratamentos em que o fluxo em massa não foi

Tabela 1. Características físicas e químicas das amostras coletadas na camada de 0 a $20 \mathrm{~cm}$ dos solos de Viçosa, Paracatu e Lassance.

\begin{tabular}{|c|c|c|c|}
\hline Características & Viçosa & Paracatu & Lassance \\
\hline Areia grossa $\left(\mathrm{g} \mathrm{kg}^{-1}\right)$ & 140 & 10 & 210 \\
\hline Areia fina $\left(\mathrm{g} \mathrm{kg}^{-1}\right)$ & 80 & 10 & 290 \\
\hline Silte $\left(\mathrm{g} \mathrm{kg}^{-1}\right)$ & 110 & 140 & 220 \\
\hline Argila $\left(\mathrm{g} \mathrm{kg}^{-1}\right)$ & 670 & 840 & 280 \\
\hline Equivalente de umidade $\left(\mathrm{kg} \mathrm{kg}^{-1}\right)$ & 0,318 & 0,298 & 0,217 \\
\hline \multicolumn{4}{|l|}{ Água retida $\left(\mathrm{kg} \mathrm{kg}^{-1}\right)$} \\
\hline$-5 \mathrm{kPa}$ & 0,48 & 0,38 & 0,28 \\
\hline$-10 \mathrm{kPa}$ & 0,37 & 0,33 & 0,26 \\
\hline$-40 \mathrm{kPa}$ & 0,34 & 0,32 & 0,20 \\
\hline$-100 \mathrm{kPa}$ & 0,30 & 0,30 & 0,16 \\
\hline Carbono orgânico $\left(\mathrm{g} \mathrm{kg}^{-1}\right)$ & 13 & 26 & 11 \\
\hline pH em água & 4,9 & 5,1 & 5,0 \\
\hline $\mathrm{Al}^{3+}\left(\mathrm{cmol}_{\mathrm{c}} \mathrm{dm}^{-3}\right)$ & 0,63 & 0,36 & 0,00 \\
\hline $\mathrm{Ca}^{2+}\left(\mathrm{cmol}_{\mathrm{c}} \mathrm{dm}^{-3}\right)$ & 0,20 & 0,30 & 4,67 \\
\hline $\mathrm{Mg}^{2+}\left(\mathrm{cmol}_{\mathrm{c}} \mathrm{dm}^{-3}\right)$ & 0,10 & 0,10 & 0,93 \\
\hline $\mathrm{K}\left(\mathrm{mg} \mathrm{dm}^{-3}\right)$ & 9,0 & 41,0 & 71,6 \\
\hline $\mathrm{P}\left(\mathrm{mg} \mathrm{dm}^{-3}\right)$ & 0,2 & 0,0 & 5,1 \\
\hline $\mathrm{S}\left(\mathrm{mg} \mathrm{dm}^{-3}\right)^{(1)}$ & 5,0 & 1,2 & 1,4 \\
\hline $\operatorname{Mn}\left(\mathrm{mg} \mathrm{dm}^{-3}\right)$ & 1,2 & 4,0 & 230,0 \\
\hline $\mathrm{Fe}\left(\mathrm{mg} \mathrm{dm}^{-3}\right)$ & 61.8 & 45,4 & 35,7 \\
\hline
\end{tabular}

${ }^{(1)}$ Extrator $\mathrm{Ca}\left(\mathrm{H}_{2} \mathrm{PO}_{4}\right)_{2}, 500 \mathrm{mg} \mathrm{L}^{-1}$ de $\mathrm{P}$ em HOAc $2 \mathrm{~mol} \mathrm{~L}^{-1}$. 
capaz de satisfazer a demanda de $\mathrm{S}$ das plantas, o mecanismo de difusão complementou seu suprimento.

Os valores obtidos do fluxo em massa e do conteúdo de $\mathrm{S}$ foram ajustados por meio de equações de regressão (Figura 1) às doses desse nutriente aplicadas. Como a difusão complementa a quantidade transportada por fluxo em massa, sua contribuição não foi quantificada diretamente, e sim, pela diferença entre o conteúdo de $\mathrm{S}$ na planta e a quantidade desse nutriente potencialmente transportada por fluxo em massa.

$\mathrm{Na}$ análise de regressão, foram eliminados os dados de conteúdo de $\mathrm{S}$, correspondentes à dose de $0 \mathrm{mg} \mathrm{dm}^{-3}$ de $\mathrm{S}$ no solo de Lassance, por fugirem à tendência observada nas demais doses.

O conteúdo de $\mathrm{S}$ das plantas aumentou em resposta às doses desse nutriente aplicadas, com exceção das plantas cultivadas no solo de Viçosa, onde não houve efeito dos tratamentos.

O fluxo em massa predominou em quase todos os tratamentos e foi o principal mecanismo de suprimento de $\mathrm{S}$ às raízes, o que corrobora os resultados de Ende (1973), Gregory et al. (1979) e Silva et al. (1998).

No solo de Viçosa, o fluxo em massa foi responsável por todo o $\mathrm{S}$ transportado até a superfície das raízes (Figura 1). A difusão não se fez presente, por causa da elevada concentração de S na solução desse solo.

As quantidades de $\mathrm{S}$ supridas por fluxo em massa aumentaram linearmente com a aplicação de doses crescentes desse nutriente no solo de Viçosa. A elevada concentração de S obtida na solução desse solo, aliada à disponibilidade de água, mantida em torno de $-10 \mathrm{kPa}$ durante todo o experimento, possibilitariam um acúmulo de $\mathrm{S}$ na superfície das raízes, devido ao fluxo em massa. Outros nutrientes transportados por fluxo em massa, como $\mathrm{Ca}$ e $\mathrm{Mg}$ (Strebel \& Duynisveld, 1989), também podem se acumular na superfície das raízes. Esses resultados também confirmam as observações de Gregory et al. (1979), que sugerem um acúmulo de S, Ca, Mg e Na em torno das raízes do trigo, decorrente do transporte por fluxo em massa.

Como a quantidade de $\mathrm{S}$ transportada até a superfície das raízes foi elevada, uma maior absorção aumentaria a sua concentração na planta sem incremento na produção de matéria seca, caracterizando um consumo de luxo. Bissani (1985) também constatou consumo de luxo de $\mathrm{S}$ em plantas de colza cultivadas em solos do Rio Grande do Sul, sob ampla variação dos teores de $\mathrm{S}$ disponível. Agrawal \& Mishra (1994) também observaram um estreitamento

Tabela 2. Concentração de enxofre na solução do solo (SSOL), enxofre potencialmente transportado por fluxo em massa (FM) e conteúdo de enxofre (CS) das plantas de soja, em função das doses de S aplicadas em amostras dos solos de Viçosa, Paracatu e Lassance.

\begin{tabular}{|c|c|c|c|c|c|}
\hline \multirow[t]{2}{*}{ Variável } & \multicolumn{5}{|c|}{ Doses de $\mathrm{S}\left(\mathrm{mg} \mathrm{dm}^{-3}\right)$} \\
\hline & 0 & 20 & 40 & 80 & 160 \\
\hline \multicolumn{6}{|c|}{ Solo de Viçosa } \\
\hline $\operatorname{SSOL}^{(1)}\left(\mathrm{mg} \mathrm{L}^{-1}\right)$ & 25,2 & 35,5 & 44,0 & 80,3 & 140,4 \\
\hline $\mathrm{FM}\left(\mathrm{mg}\right.$ vaso $\left.^{-1}\right)$ & 70,3 & 92,6 & 112,3 & 244,4 & 336,7 \\
\hline $\mathrm{CS}\left(\mathrm{mg} \mathrm{vaso}^{-1}\right)$ & 24,5 & 22,7 & 24,0 & 27,9 & 24,4 \\
\hline \multicolumn{6}{|c|}{ Solo de Paracatu } \\
\hline $\operatorname{SSOL}^{(1)}\left(\mathrm{mg} \mathrm{L}^{-1}\right)$ & 3,9 & 9,0 & 15,4 & 39,0 & 101,1 \\
\hline $\mathrm{FM}\left(\mathrm{mg} \operatorname{vaso}^{-1}\right)$ & 13,8 & 32,0 & 51,9 & 154,9 & 409,3 \\
\hline $\mathrm{CS}\left(\mathrm{mg} \mathrm{vaso}^{-1}\right)$ & 24,2 & 29,7 & 32,0 & 37,3 & 39,2 \\
\hline \multicolumn{6}{|c|}{ Solo de Lassance } \\
\hline $\operatorname{SSOL}^{(1)}\left(\mathrm{mg} \mathrm{L}^{-1}\right)$ & 3,0 & 5,8 & 11,2 & 28,0 & 80,3 \\
\hline FM (mg vaso $\left.{ }^{-1}\right)$ & 10,4 & 16,8 & 36,0 & 92,0 & 329,2 \\
\hline $\mathrm{CS}\left(\mathrm{mg}\right.$ vaso $\left.^{-1}\right)$ & 32,5 & 27,4 & 30,7 & 32.7 & 41.8 \\
\hline
\end{tabular}

(1)Extrato de pasta de saturação. 
na relação N/S nas folhas de soja, que não foi proporcional ao aumento na produção de matéria seca ou de grãos, constituindo, assim, consumo de luxo de enxofre. Embora existam evidências de consumo
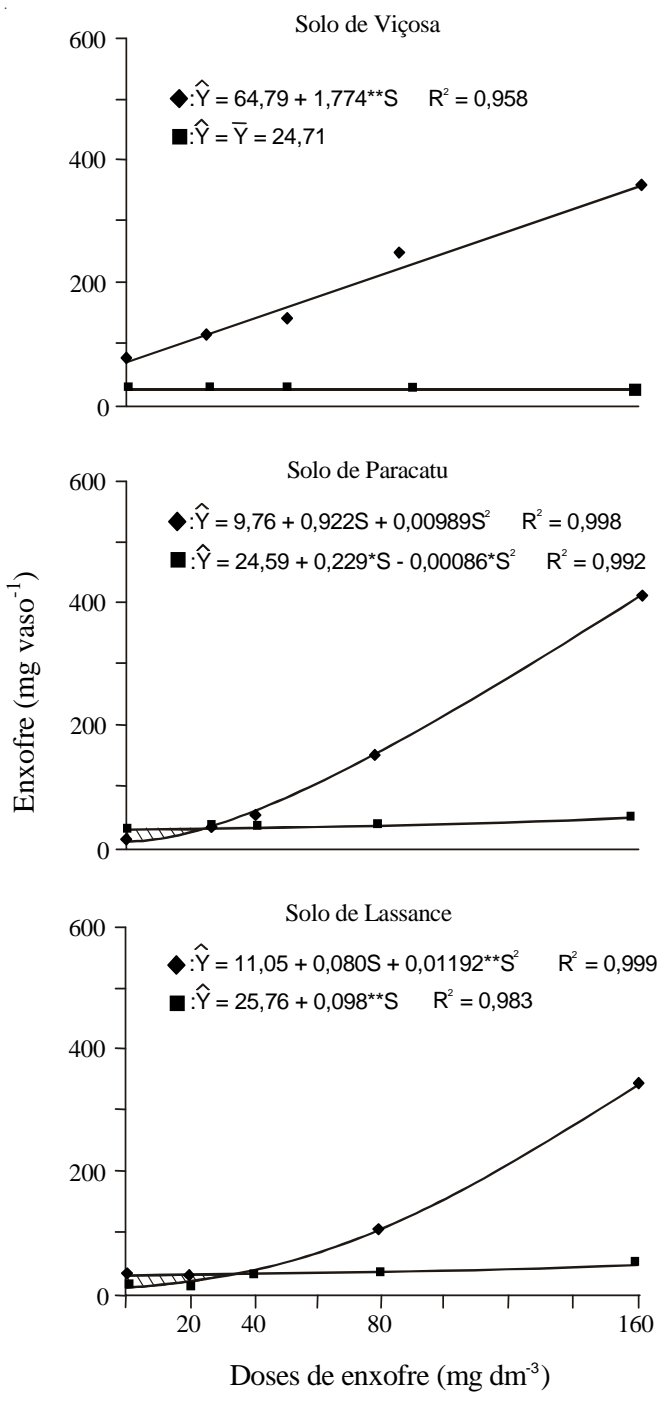

Figura 1. Enxofre potencialmente transportado por fluxo em massa até a superfície das raízes $(\bullet)$ e conteúdo de enxofre (థ) das plantas de soja, em função das doses de $S$ aplicadas em amostras dos solos de Viçosa, Paracatu e Lassance. Nas amostras dos solos de Paracatu e Lassance a parte com hachuras corresponde ao $\mathrm{S}$ transportado por difusão. de luxo, a concentração de $\mathrm{S}$ nas plantas de soja aumentou na mesma proporção que a matéria seca, permitindo sugerir que o excesso de $\mathrm{S}$ transportado por fluxo em massa tenha se acumulado ou precipitado na superfície das raízes.

A precipitação de $\mathrm{S}$ deve ocorrer principalmente na forma de sulfato de cálcio. Barber et al. (1963) publicaram uma auto-radiografia mostrando o acúmulo de ${ }^{35} \mathrm{~S}$ sobre uma raiz de milho. $\mathrm{O}$ acúmulo próximo à superfície das raízes, semelhante a uma contra-difusão, sugere a ocorrência de precipitação de sulfato de cálcio (Barber, 1984). Malzer \& Barber (1975) demonstraram que o sulfato de cálcio pode precipitar-se próximo às raízes tanto nas plantas cultivadas no solo quanto nas cultivadas em solução nutritiva saturada com sulfato de cálcio. A solubilidade do sulfato de cálcio em água a $25^{\circ} \mathrm{C}$ é de $15,4 \mathrm{mmol} \mathrm{L}^{-1}$. Esta concentração pode ser facilmente atingida quando o fluxo em massa transporta $\mathrm{Ca}$ e $\mathrm{S}$ para a superfície das raízes e a absorção de água é mais rápida que a de qualquer um desses elementos (Barber, 1984).

Nos solos de Paracatu e Lassance, a contribuição do fluxo em massa aumentou, em resposta às doses de $\mathrm{S}$ aplicadas, e se ajustou a equações quadráticas (Figura 1).

Nesses solos, tanto o fluxo em massa quanto a difusão participaram do transporte de $\mathrm{S}$, até que as quantidades supridas se igualassem às absorvidas pela planta (Figura 1). A partir desse ponto, o fluxo em massa passou a suprir a totalidade de S, e o excedente se acumulou e, ou, precipitou na superfície das raízes, à semelhança do que ocorreu no solo de Viçosa. As doses necessárias para atingir tal ponto foram estimadas em 16,9 e $35,9 \mathrm{mg} \mathrm{dm}^{-3}$ de $\mathrm{S}$ nos solos de Paracatu e Lassance, respectivamente. Substituindo-se esses valores nas equações de $\mathrm{S}$ na solução do solo, em função das doses desse nutriente aplicadas (Tabela 3), foram obtidas as concentrações de S na solução do solo necessárias para que a quantidade potencialmente transportada por fluxo em massa fosse igual àquela que foi absorvida e acumulada pela planta.

O processo de difusão participou do suprimento de $S$ às raízes quando a sua concentração estava abaixo de 8,2 e 10,3 $\mathrm{mg} \mathrm{L}^{-1}$ na solução dos solos de Paracatu e Lassance, respectivamente. 
Estabelecida a dose mínima necessária em cada solo para atingir o ponto em que o $\mathrm{S}$ absorvido pela planta passa a ser transportado exclusivamente por fluxo em massa, determinou-se a contribuição porcentual do fluxo em massa e da difusão para o transporte de $\mathrm{S}$ até as raízes abaixo desse valor de concentração. Como os dois mecanismos atuam de forma complementar até atingir tal ponto, foram analisados apenas os dados referentes ao fluxo em massa. A contribuição da difusão pode ser obtida subtraindo-se de 100 a porcentagem de $\mathrm{S}$ transportado por fluxo em massa.

Ajustaram-se equações quadráticas no solo de Lassance, e linear no solo de Paracatu (Figura 2). Na dose $0 \mathrm{mg} \mathrm{dm}^{-3}$ de $\mathrm{S}$, o fluxo em massa transportou apenas 39,2 e 42,7 \% do $\mathrm{S}$ absorvido pelas plantas nos solos de Paracatu e Lassance, respectivamente.

Tabela 3. Equações de regressão para concentração de enxofre na solução do solo, em $\mathrm{mg} \mathrm{L}^{-1}$, em função das doses de $\mathrm{S}$ aplicadas, em $\mathrm{mg} \mathrm{dm}^{-3}$, em amostras dos solos de Viçosa, Paracatu e Lassance.

\begin{tabular}{clc}
\hline Solo & \multicolumn{1}{c}{ Equação de regressão } & $\mathrm{R}^{2}$ \\
\hline Viçosa & $\hat{\mathrm{Y}}=20,67+0,74 * * \mathrm{~S}$ & 0,993 \\
Paracatu & $\hat{\mathrm{Y}}=3,26+0,2517 * * \mathrm{~S}+0,00226 * * \mathrm{~S}^{2}$ & 0,999 \\
Lassance & $\hat{\mathrm{Y}}=2,59+0,1373 * * \mathrm{~S}+0,00218 * * \mathrm{~S}^{2}$ & 0,999 \\
\hline
\end{tabular}

**Significativo a $1 \%$ de probabilidade.

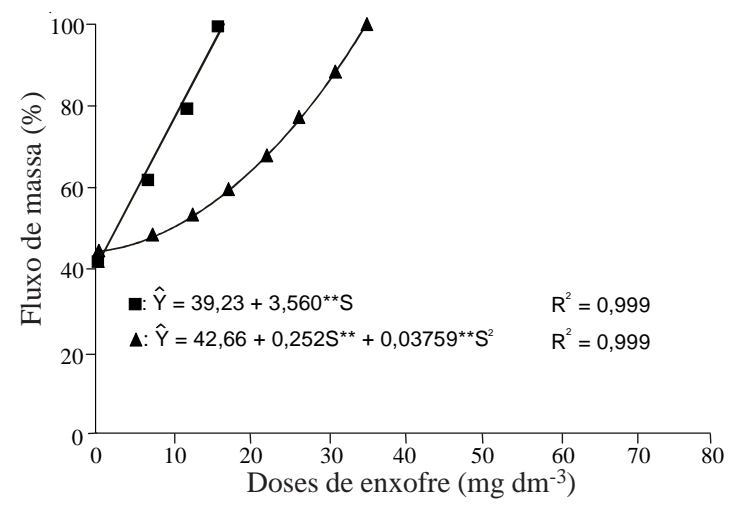

Figura 2. Contribuição porcentual do fluxo em massa na absorção de S por plantas de soja, em função das doses de $\mathrm{S}$ aplicadas em amostras dos solos de Paracatu (a) e Lassance ( $\mathbf{\Delta})$.
Depreende-se que o transporte de S para as raízes depende não somente do fluxo em massa, mas também da difusão, quando a concentração do nutriente na solução do solo estiver abaixo desses valores. O suprimento de outros nutrientes que podem ser transportados exclusivamente por fluxo em massa quando existe uma concentração adequada no solo, também é complementado pelo processo de difusão quando esses nutrientes estão em baixa concentração na solução do solo. Isso foi observado com relação ao N (Liao \& Bartholomew, 1974; Okajima \& Taniyama, 1980; Strebel \& Duynisveld, 1989), Ca (Prenzel, 1979) e Mg (Al-Abbas \& Barber, 1964; Vargas et al., 1983).

\section{Conclusões}

1. O fluxo em massa é o principal mecanismo de transporte de enxofre para a superfície radicular da soja.

2. Em alta concentração de enxofre na solução do solo, o fluxo em massa supre quantidades de enxofre superiores às absorvidas pela planta.

3. A contribuição da difusão para o suprimento de enxofre ocorre apenas em baixa concentração desse nutriente na solução do solo.

\section{Referências}

AGRAWAL, H. P.; MISHRA, A. K. Sulphur nutrition of soybean. Communications in Soil Science and Plant Analysis, New York, v. 25, n. 9/10, p. 1303-1312, 1994.

AL-ABBAS, H.; BARBER, S. A. Effects of root growth and mass-flow on the availability of soil calcium and magnesium to soybeans in a greenhouse experiment. Soil Science, Baltimore, v. 97, n. 1/6, p. 103-107, Jan./June 1964.

ALVAREZ VENEGAS, V. H. Equilíbrio de formas disponíveis de fósforo e enxofre em dois Latossolos de Minas Gerais. 1974. 125 f. Dissertação (Mestrado) - Universidade Federal de Viçosa, Viçosa. 1974.

BARBER, S. A. Soil nutrient bioavailability: a mechanist approach. New York: J. Wiley, 1984. 398 p.

BARBER, S. A.; WALKER, J. M.; VASEY, E. H. Mechanisms for the movement of plant nutrients from the soil and fertilizer to the plant root. Journal of Agricultural Food and Chemistry, Washington, v. 11, n. 3, p. 204-207, May/June 1963. 
BISSANI, C. A. Disponibilidade de enxofre para as plantas em solos do Rio Grande Sul. 1985. 198 f. Dissertação (Mestrado) - Universidade do Rio Grande do Sul, Porto Alegre. 1985.

BLANCHAR, R. W.; REHM, G; CALDWELL, A. C. Sulfur in plant material by digestion with nitric and perchloric acid. Soil Science Society of America Proceedings, Madison, v. 29, n. 1, p. 71-72, Jan./Feb. 1965.

CHESNIN, L.; YIEN, C. H. Turbidimetric determination of available sulfates. Soil Science Society of America Proceedings, Madison, v. 15, p. 149-151, 1950.

COMISSÃO DE FERTILIDADE DO SOLO DO ESTADO DE MINAS GERAIS (Lavras, MG). Recomendação para o uso de corretivos e fertilizantes em Minas Gerais: $4^{\mathrm{a}}$ aproximação. Lavras, 1989. 176 p.

EMBRAPA. Centro Nacional de Pesquisa de Solos (Rio de Janeiro, RJ). Manual de métodos de análise de solos. 2. ed. rev. atual. Rio de Janeiro, 1997. 212 p.

ENDE, J. van den. Extraction methods for the determination of major elements in greenhouse soils and pottingand culture media. Acta Horticulturae, The Hague, v. 29, p. 125-139, 1973.

GREGORY, P. J.; CRAWFORD, D. V.; McGOWAN, M. Nutrient relations of winter wheat. 2. Movement of nutrients to the root and their uptake. Journal of Agricultural Science, Part 2, Cambridge, v. 93, p. 495-504, Oct. 1979.

LIAO, C. F. H.; BARTHOLOMEW, W. V. Relation between nitrate absorption and water transpiration by corn. Soil Science Society of America Proceedings, Madison, v. 38, n. 3, p. 472-477, May/June 1974.

MALZER, G. L.; BARBER, S. A. Precipitation of calcium and strontium sulfates around plant roots and its evaluation. Soil Science Society of America Proceedings, Madison, v. 39, n. 3, p. 492-495, May/June 1975.

OKAJIMA, H.; TANIYAMA, I. Significance of mass flow in nitrate-nitrogen supply to plant roots. Soil Science and Plant Nutrition, Tokyo, v. 26, n. 3, p. 363-374, 1980.
OLIVER, S.; BARBER, S. A. An evaluation of the mechanisms governing the supply of $\mathrm{Ca}, \mathrm{Mg}, \mathrm{K}$ and $\mathrm{Na}$ to soybean roots (Glycine max). Soil Science Society of America Proceedings, Madison, v. 30, n. 1, p. 82-86, Jan./ Feb. 1966.

PATEL, C. L.; GHILYAL, B. P.; TOMAR, V. S. Nutrient flow rates in rice roots under varying drainage conditions. Plant and Soil, The Hague, v. 77, n. 2/3, p. 243-252, 1984.

PRENZEL, J. Mass flow to the root system and mineral uptake of a beech stand calculated from 3-year field data. Plant and Soil, The Hague, v. 51 n. 1, p. 39-49, 1979.

RUIZ, H. A. Efeito do conteúdo de água sobre o transporte de fósforo em dois Latossolos. 1986. 86 f. Tese (Doutorado) - Universidade Federal de Viçosa, Viçosa. 1986.

SILVA, D. J.; ALVAREZ VENEGAS , V. H.; RUIZ, H. A. Fluxo em massa e difusão de enxofre para raízes de milho em solos ácidos de Minas Gerais. Revista Brasileira de Ciência do Solo, Viçosa, MG, v. 22, n. 1, p. 109-114, jan./ mar. 1998.

STREBEL, O.; DUYNISVELD, W. H. M. Nitrogen supply to cereals and sugar beet by mass flow and diffusion on silty loam soil. Zeitschrift fuer Pflanzenernaehrung und Bodenkunde, Weinheim, v. 152, p. 135-141, 1989.

VARGAS, R. M. B.; MEURER, E. J.; ANGHINONI, I. Mecanismos de suprimento de fósforo, potássio, cálcio e magnésio às raízes de milho em solos do Rio Grande do Sul. Revista Brasileira de Ciência do Solo, Campinas, v. 7, n. 2, p. 143-148, maio/ago. 1983.

XU, M. G.; ZHANG, Y. P.; SUN, B. H. Mechanisms for the movement of $\mathrm{Fe}, \mathrm{Mn}, \mathrm{Cu}$, and $\mathrm{Zn}$ to plant roots in Loessal Soil and Lou Soil. Pedosphere, Beijing, v. 6, n. 3 , p. 245-254, 1996a.

XU, M. G.; ZHANG, Y. P.; ZHANG, J. C.; SUN, B. H. Mechanisms for the movement of $\mathrm{Ca}, \mathrm{Mg}, \mathrm{P}$ and $\mathrm{K}$ to plant roots growing in two soils. Scientia Agricultura Sinica, [China], v. 29, n. 5, p. 76-82, 1996 b. 